\title{
FAUT-IL ÉCOUTER POUR ENTENDRE ?
}

\author{
Andrée Chauvin-Vileno ${ }^{1}$
}

Deux modalités de narration radiophonique, celle du documentaire (en l'occurrence Les Pieds sur Terre sur France-Culture) et celle de la matinale sont mises en regard à propos de l'expérience de détention de Clotilde Reiss en Iran (2009-2010). Alors que la matinale scénarise, démultiplie angles et temps, et fait fond sur la doxa, le documentaire use du montage pour ménager au témoignage son identité énonciative et son mouvement propre, et rendre sensible la singularité vécue de la réalité.

Dans l'univers sémiotique de la radio $^{2}$, le rapport sensible au medium participe du rapport " sensé » au monde que construisent les discours. Prenant ses formes au sein du paysage sonore lui-même et composant (avec) les genres radiophoniques, le narratif traverse ou structure ces discours.

1 Andrée Chauvin-Vileno est professeur à l'Université de Franche Comté.

2 La radio se définit par une présence sensible d'une forte densité paradoxale puisque sans image et sans espace et elle instaure une temporalité particulière comme l'ont analysé notamment Schaeffer et Tétu. Cf. aussi Charaudeau (1984 et 2005).

Recherches en communication, $\mathrm{n}^{\circ} 37$ (2012). 
Parmi les missions que s'assigne la radio, comprendre le monde n'est pas la moindre : entendre pour entendre ${ }^{1}$.Comment la narration radiophonique donne-t-elle à entendre ? Notre hypothèse est que la radio (ou plutôt les radios) proposent des régimes d'intelligibilité qui procèdent de modalités de narration et de postures de réception différentes.

Ces modalités de narration seront explorées ici à partir de récits (ou d'éléments de récits) qui se rattachent à l'histoire de Clotilde Reiss. Emprisonnée lors de la période de contestation massive et de répression brutale en Iran, après la réélection du président Ahmaninedjad en juin 2009, puis assignée à résidence, la jeune étudiante française dont le procès avait été largement médiatisé, revient en France le 16 mai 2010. Les déclarations et les commentaires dans les médias mettent alors selon les cas au premier plan les scénarios du marchandage diplomatique ou de l'espionnage, les inquiétudes sur l'Iran, la dénonciation de la répression.

La série de Les Pieds sur terre consacrée, un an après, à cette répression en Iran, et dont deux émissions portent le témoignage de C. Reiss, un mois après le retour en France de celle-ci, a été l'élément déclencheur du présent travail. Le contraste est net entre ce documentaire et le traitement standard du discours d'information des principaux organes de presse (écrite, télévisée et radiophonique) qui en ont pourtant relayé la diffusion. Etre auditeur d'un témoignage dans une émission qui rend possible d'écouter ne revient pas à entendre les nouvelles, et permet de les comprendre autrement.

Ce n'est pas à une comparaison que nous nous livrerons ici mais à une mise en regard, en nous référant à un double corpus, des extraits de la matinale du 17 mai sur France-Inter au lendemain du retour de $\mathrm{C}$ Reiss, et ce documentaire quotidien au style bien marqué, Les Pieds sur terre (France- Culture) des 10 et 11 juin $2010^{2}$.

1 Le TLF en ligne (http://atilf.atilf.fr/) distingue trois acceptions principales pour ce verbe : domaines de l'audition, de l'intellection, de la volition.

2 La matinale n'est plus disponible à l'écoute mais les textes des chroniques sont consultables sur le site de France-Inter.

Les deux émissions de Les Pieds sur terre (juin 2010) ne sont plus disponibles à l'écoute sur le site mais à cette adresse : http://www.touslespodcasts. com/annuaire/ radio-tv/radio-nationales/833-11

Leur transcription est accessible : http://www.iranfocus.com/fr/ index.php? option $=$ com_content\&view $=$ article \&id=8078:iran-le-temoignage-de-clotilde-reisssur-france-culture-transcription-\&catid=17:la-vie-en-iran \&Itemid $=41$ 


\section{Une nouvelle, deux émissions, deux genres radiophoniques}

Une matinale se définit à la fois par sa tranche horaire et par la dominante thématique de l'information à laquelle elle est dévolue principalement, avec le retour toutes les heures ou demi-heures des bulletins puis des journaux plus développés, et des rubriques régulières telles que les séquences d'interview avec un invité, les chroniques diverses, selon « une alternance très significative entre la sécheresse de l'information, réductible au flash, et les multiples billets, chroniques et éditoriaux » (Tétu, 1994).

En mai 2010, sur France-Inter la matinale est (encore) animée par Nicolas Demorand ${ }^{1}$ (du lundi au vendredi) et elle porte le nom de sa tranche (Le Six trente Dix). Le retour en France de C Reiss, sans être le sujet principal de la matinale du 17 mai, en est l'un des fils conducteurs repris sous des angles différents d'une part dans les journaux, d'autre part dans certaines chroniques (billet politico-économique, revue de presse $)^{2}$.

L'émission quotidienne Les Pieds sur terre, produite par Sonia Kronlund depuis $2002^{3}$, est programmée de $13 \mathrm{~h} 30$ à $14 \mathrm{~h}$ (du lundi au

1 N. Demorand producteur de la matinale de France-Culture (2002-2006) puis de celle de France-Inter (2006-2010) a quitté Radio-France pour Europe 1 à l'automne 2010, avant de devenir directeur du quotidien Libération.

2 L'annonce du retour de C. Reiss est présente dans tous les flashes et fait l'ouverture du bulletin de $8 \mathrm{~h}$ au cours duquel on peut réécouter une interview de J.-L. Bianco (député socialiste) par Demorand diffusée avant $7 \mathrm{~h}$.

La thématique dominante de la matinale est économique avec pour invité J.-P. Jouyet, président de l'Autorité des marchés financiers.

La chronique géopolitique de Bernard Guetta (http://www.franceinter.fr/chro/ geopolitique/91694) intitulée "Les bons offices turco-brésiliens » s'ouvre sur les négociations pour la libération de C. Reiss, comme élément d'un accord d'ensemble sur lequel il se centre, accord entre l'Iran, la Turquie et le Brésil en matière de combustibles nucléaires.

La revue de presse de Bruno Duvic (http://www.franceinter.fr/chro/

larevuedepresse/91646), intitulée "Clotilde Reiss libre : les questions » lui est consacrée pour un bon tiers, après une entrée en matière sur une joueuse de tennis franco-iranienne à Roland Garros, qui amène le revuiste à la désigner ainsi : une « autre Française très liée à l'Iran ». La fin de la séquence Reiss est amenée par une transition tout aussi acrobatique sur la discrétion de N. Sarkozy (« On est loin du battage médiatique organisé autour de libération des infirmières bulgares, des cadres de l'Arche de Zoé ou de l'accueil en fanfare d'Ingrid Betancourt. ») qui est ensuite réputée s'exercer aussi en matière de réforme de retraites.

3 S. Kronlund a publié chez Actes Sud en février 2012 Les Pieds sur terre. Nouvelles 
vendredi). Elle est présentée sur le site de France-Culture comme « une demi-heure quotidienne de documentaire pour cerner au plus près la réalité de la vie moderne » (2008), de « reportage sans commentaire » pour « aller vers ceux qui se battent» et « tenter d'écouter sans analyser ceux dont on commente abondamment les faits et gestes, aller sur leur terrain et y rester » (2010) ${ }^{1}$. Ces présentations plus ou moins développées insistent sur la relation au terrain et au temps («prendre le temps»), sur le prix accordé à la parole comme alternative au commentaire et à l'analyse suspectés de s'approprier l'expérience. Si le commentaire est réputé absent, le point de vue est affirmé : " aller vers ceux qui luttent ». Le terme de témoignage n'est pas employé pour la présentation générale (il l'est pour les deux émissions qui nous intéressent), mais deux désignateurs de genre apparaissent, reportage et documentaire alors que la forme de l'entretien est suggérée par " écouter».

Les Pieds sur terre sont une tentative de radio-réalité ${ }^{2}$ qui fait fond essentiellement sur la parole des anonymes, des locuteurs ordinaires « les gens, le peuple, pas les people »3. Ce n'est pas le cas de C Reiss, très exposée médiatiquement et dont le témoignage est présenté par d'autres organes de presse comme en exclusivité, participant ainsi de la valorisation du scoop.

Par exemple sur le site de Paris Match.com en date du 10 juin on peut voir la Une titrée « Clotilde Reiss. Ses 47 jours à la prison d'Evin » avec une photo couleur prise lors du procès ${ }^{4}$ et lire :

Pour la première fois [...] Clotilde Reiss se confie sur son mois et demi en détention en Iran. [...] Elle est la seule Française à avoir séjourné à la prison d'Evin. [...] elle a choisi une journaliste qu'elle connaît, Sonia Kronlund [...] pour se livrer, une fois pour toute, lors d'un long entretien assez détaillé. Cette inter-

$d u$ réel, un ouvrage reprenant quarante-quatre histoires (http://www.actes-sud.fr/ catalogue/societe/les-pieds-sur-terre).

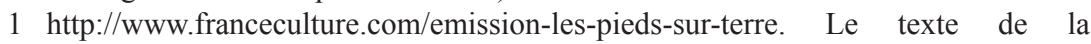
présentation est un peu différent en 2012. Les archives recensent les émissions à partir de 2005)..

2 Expression empruntée à François Jost, http://comprendrelatele.blog.lemonde. $\mathrm{fr} / 2010 / 11 / 06$

3 Télérama http://www.telerama.fr/divers/19259_petite_mere_du_peuple.php

4 http://www.parismatch.com/Actu-Match/Monde/Actu/Clotilde-Reiss.-Ses-47jours-a-la-prison-d-Evin-192004/ 
view sera diffusée [...]ce jeudi et demain, de $13 \mathrm{~h} 30$ à $14 \mathrm{~h} 00$ sur France Culture, mais déjà plusieurs extraits circulent. ${ }^{1}$

Néanmoins la programmation de l'entretien sur France-Culture s'inscrit d'une part dans le sillage du travail de longue date de la productrice sur l'Iran, d'autre part dans une série courant sur la semaine, selon un principe d'organisation récurrent. Les deux émissions qui racontent «L'histoire de Clotilde Reiss, ancienne détenue dans les prisons d'Iran » (10 et 11 juin) sont précédées par un terrible « Récits de détention 》 (9 juin) et par deux autres témoignages de femmes actrices de la contestation, une étudiante iranienne vivant désormais à Paris (« Manifestante verte ») et une bloggeuse ( « Soheila révolutionnaire cybernétique ») (7 et 8 juin). Si C. Reiss, loin d'être une anonyme s'est trouvée projetée malgré elle dans une dimension politique et sur une scène médiatique, le documentaire la rend à sa vérité de personne.

\section{Dire autrement, faire (entendre) autre chose}

Comment les deux types d'émission abordent-elles la narration ? Qui raconte ? Comment apparaît le récit ? Quels en sont les protagonistes ? Quelle relation au réel et à l'auditeur ces caractéristiques construisent-elles?

« Raconter » ou plutôt « faire raconter » est l'objectif explicite du documentaire de France Culture. Ce n'est pas celui de la matinale, dans la mesure où une séquence d'entretien n'est pas consacrée à une telle relation, et où le discours d'information n'affiche pas comme but en soi de « raconter l'actualité ». Décrire et raconter sont mis au service d'expliquer et commenter (Charaudeau, 2005).

L'émission de S. Kronlund offre à C. Reiss un espace de parole qu'elle occupe presque entièrement, en donnant l'impression à l'auditeur qu'elle organise son dire. L'introduction de la productrice cadre certes l'émission, mais passé ce seuil, C. Reiss jouit d'une certaine autonomie, pour suivre son projet de mémoire, dont la modalité dominante n'est pas l'émergence spontanée du souvenir, mais la volonté d'exposer une expérience en optant pour des jalons (tel ou tel moment)

1 Libération du 10 juin publie un article de J.-P. Perrin « Clotilde Reiss, croquis de prison » qui comporte des extraits de l'entretien, des dessins auxquels la jeune femme fera référence et annonce l'émission. http://www.liberation.fr/monde/0101640510clotilde-reiss-croquis-de-prison 
et des étais (dessins et documents). Cette narration n'est pas monologale, la journaliste (qui la tutoie) pose des questions à C. Reiss, faisant état de certaines connaissances, sans conduire pour autant l'entretien. Plus qu'une interview, il semble s'agir d'une conversation privée sur un sujet d'intérêt général, comme si les questions surgissaient au fil d'un échange interpersonnel.

La matinale juxtapose, emboîte et enchaîne un grand nombre de discours émanant d'un grand nombre de locuteurs différents sous l'impulsion « orchestrante » du producteur-animateur et, par délégation, des journalistes présentateurs des bulletins et des chroniqueurs. On n'entend le discours rapporté de C. Reiss (sous la modalité de la reformulation ou de la citation sonore directe) que pour des extraits de sa déclaration publique de la veille à l'Elysée où elle remercie le chef de l'Etat et tous ceux qui ont contribué à sa libération. Les autres relais citationnels font entendre des personnalités politiques, des exégètes ou des experts, des journalistes, des séquences d'autres émissions y compris de la même station, archivées ou toutes récentes (l'heure précédente). Le discours radiophonique de la matinale en particulier se construit ainsi en " patchwork de petites unités bariolées » (Mouillaud, 1984, p. 68) sur les plans énonciatif, vocal et générique, tandis que celui du documentaire le fait autour d'un locuteur principal, centre de l'intérêt.

Dans le documentaire, une interlocutrice (S. Kronlund) fait le lien entre la narratrice et l'auditoire, elle recueille son récit et pose des questions à C. Reiss, mais dans la matinale, il est question de C. Reiss et des questions sont suscitées à partir de son cas ${ }^{1}$.

Alors que le récit autodiégétique du documentaire est essentiellement rétrospectif, les assertions et les interrogations des bulletins d'information sont multidirectionnelles, comme s'il s'agissait autant d'embrasser ce qui s'est passé que d'anticiper sur des développements à venir, afin de faire ressentir la saisie d'un présent qui advient sans arrêt, qui rebondit, dans un « vertige du vite» (Castant, 1995), que cultivent mimétiquement les médias de flux en particulier et le discours d'information en général (Tétu, 1994).

Certes le journaliste qui présente les bulletins déclare bien : « On en sait un peu plus sur ce que $\mathrm{C}$. Reiss a vécu pendant ces dix mois » mais

1 Cf. le titre de la revue de presse de B. Duvic, sa phrase « Beaucoup de questions, donc, après cette libération », la forme interrogative de la transition de N. Demorand «Pourquoi les mollahs capables de la plus grande cruauté ont-ils laissé partir Clotilde Reiss ? ». 
ce n'est pas le propos essentiel de la matinale, qui explore plutôt les prolongements et l'émergence d'une « affaire » à révéler et à dénouer.

" Emprisonnée jugée condamnée puis assignée à résidence à Téhéran la Française Clotilde Reiss a regagné Paris hier libérée officiellement contre une amende de 245000 euros après dix mois de démêlés diplomatico-judiciaires. » (A. Le Gougec, ouverture du bulletin de 8h). Dans ce passage qui rappelle de manière condensée la succession des événements, le syntagme figé " démêlés diplomatico-judiciaires » ramène au connu des péripéties singulières. L'accent d'insistance sur « officiellement» (marqué ici par l'italique) laisse entendre et attendre une autre version officieuse supputée. D'ailleurs, dans un autre flash, le journaliste annonce « Le dossier C. Reiss ne se referme pas pour autant ». Ce dossier sera aussi appelé « l'affaire». Il ne s'agit pas de la destinée de C. Reiss, ni de celle des victimes silencieuses de la répression du régime, comme c'est le cas dans le documentaire mais d'un nœud d'intrigues dont les retentissements politiques sont privilégiés.

Il semble, pour reprendre les termes de Tétu que le «parti pris narratif consiste à répondre à deux questions en même temps: qu'est-ce qui est arrivé, et pourquoi ? autrement dit à mêler le quoi et le pourquoi ». Dès lors le « sens se trouve réduit à leur rapport explicatif. » (Tétu, 1994). Les causalités sont exacerbées, il s'agit de mettre au jour des ressorts, une dimension cachée : «Un ingénieur iranien soupçonné de business nucléaire [est] reparti libre pour Téhéran et bientôt l'assassin de l'ancien Premier Ministre du Chah » (revue de presse de B. Duvic), Pierre Siramhi ancien sous-directeur de la DGSE affirme que C Reiss faisait du renseignement. L'incidence de l'influence d'autres pays et la corrélation entre qualité des relations diplomatiques et épilogue de l'assignation à résidence sont analysées (notamment dans la chronique de B. Guetta). C'est une mise en réseau des événements qui est visée, soit sur un mode associatif de coïncidence presque ludique comme dans la revue de presse qui s'ouvre sur une franco-iranienne à Roland Garros pour en venir à C. Reiss, ou sur un mode explicatif qui s'inscrit sur le plan de la politique intérieure ou internationale.

A la faveur de cette mise en réseau, ce sont les personnalités connues qui sont évoquées ou convoquées, qu'il s'agisse des réactions citées ou reformulées des politiques en France (de B. Hamon à J.-M. Le Pen), ou du rôle supposé des acteurs internationaux comme dans ce passage d'un des journaux: "Les chefs d'état brésilien et sénégalais auraient joué un rôle dans la libération de $\mathrm{C}$ Reiss. Lula roi des médiateurs !» On le constate ensuite dans ce passage, le principe d'explica- 
tion à peine dégagé, le discours passe à autre chose comme poussé par cette même dynamique et c'est Lula, son passé de syndicaliste supposé expliquer à son tour son sens des négociations, qui va faire l'objet d'un développement.

Les protagonistes sont donc bien moins Clotilde et ses compagnons de prison, ou le peuple iranien, que ceux, bien connus, de la scène nationale et internationale. D'autre part le mode de relation privilégié est celui de la controverse, comme une réfraction par le discours d'information du débat public. On le perçoit à la mention d'opinions contradictoires ou divergentes, notamment par les discours rapportés des locuteurs (citations sonores dans les bulletins, citations reprises ou reformulations des organes de la presse écrite dans la revue de presse) et à la caractérisation métadiscursive explicite : « La polémique s'est enflammée à peine annoncée la nouvelle de la libération » de la jeune femme, signale un bulletin.

Contrairement au documentaire, la matinale ne délivre pas un récit suivi sous régie unique, car elle tire un grand nombre de fils narratifs et est à la fois fragmentée et continuée. Elle s'adapte aux conditions de la prise en cours de route en sachant que les bulletins permettent une annonce-récapitulation, que la progression et la diversité des séquences assurent la possibilité d'une écoute maintenue. Tout au long de la matinale, ce sont des éclats de récit pour histoires à recomposer, une sorte de puzzle mouvant qui se reconfigure sans cesse, « qui laisse penser sans cesse que quelque chose a changé, dans ce qui se passe, et dans ce qu'on en sait. [...]Rapidité et renouvellement produisent ce sentiment d'un halètement du temps dont le rythme du matin est très symptomatique » (Tétu, 1994). Un équilibre se fait d'un bulletin à l'autre dans un agencement d'épisodes et de variations qui marquent l'avancée, tandis que les chroniques synthétisent pour donner du sens. A cet égard, la revue de presse qui se pose en surplomb apparaît comme un exercice de funambulisme ${ }^{1}$.

1 Rendre compte de l'ailleurs de la presse écrite confronte la revue de presse à une hétérogénéité énonciative et thématique. La cohérence à assurer, plus ou moins convaincante, promeut en filigrane un point de vue. Dans la revue de presse de B. Duvic, C. Reiss est présentée de façon avantageuse : « la vedette du jour [...] douceur et discrétion ». Quelques-uns des articles repris par le revuiste évoquent les conditions de vie des Iraniens, mais deux tiers des citations/reformulations tournent autour du mystère et du silence des gouvernements, sans développer toutefois la controverse. 
Le renouvellement par ouverture de perspectives (les relations internationales), par mise en doute (et si cette jeune femme était une vraie espionne ?), par annonce (la controverse ne fait que commencer), par bifurcations (Lula, Sarkozy) procède d'une sorte d'effet de suspense avec pour enjeu la promesse d'un savoir à acquérir, d'un accès à la complexité entrevue et manifestée. Tétu remarque :

Un peu à la façon dont le roman policier se nourrit non pas de la recherche de la solution, mais de son retard, l'information n'a pas pour but de dire un savoir, mais de maintenir une incertitude. Et chaque item a d'abord pour fonction de faire attendre la suite. (Tétu, 1993)

L'appétit presque déclaré pour le romanesque est suggéré par ce commentaire d'un bulletin qui fait suite à l'évocation des déclarations de l'ancien patron de la DGSE : « autant dire que ce n'est que le début d'un nouveau feuilleton ». Relèvent du feuilleton les personnages en opposition manichéenne que se plaisent à faire surgir les désignations des protagonistes : «La France n'a pas du tout envie de voir un assassin rentrer dans un pays où une jeune innocente est assignée à résidence $\rangle^{1}$, ou encore les désignations métonymiques comme « les mollahs », pour référer au gouvernement iranien ${ }^{2}$.

De telles formules invitent à reconnaître des éléments de scénarios et assurent une sorte de familiarité, comme les syntagmes figés, qui, plus qu'ils n'émaillent le discours journalistique, en constituent ici le tissu principal, comme permettent de le constater les quelques citations supra. Reconnaître la langue dans ses expressions bien établies, reconnaître des histoires déjà rencontrées permettrait une sorte d'équilibre, d'apprivoisement de la complexité et de l'incertitude, le savoir promis se nourrissant d'un savoir partagé, voire convenu.

\section{Le témoignage : montage d'un dire en devenir}

Les émissions de Les Pieds sur terre que nous considérons ne constituent pas seulement un autre traitement d'une même matière événementielle, un discours parallèle ou complémentaire des «news »

1 Ali Vakili Rad assassin de Shapour Bakthiar (ancien premier ministre du Chah) d'abord condamné à perpétuité, puis libérable en 2010 a été expulsé vers l'Iran le 18 mai 2010.

2 Voir note 1 sur la page 46. 
mais elles revendiquent le contrepoint, voire proposent un contrediscours en contraste avec ceux du paysage médiatique qui sont évoqués. Les « trente minutes sans commentaire » ne signifient pas une absence de l'instance de production bien au contraire. Celle-ci se manifeste par les choix de programmation, par l'écriture documentaire et sa pratique du montage autant que par l'explicitation des visées assumée par un «nous ». La narration que C. Reiss fait à S. Kronlund se situe dans un cadre fermement posé ${ }^{1}$ avec une forte inscription énonciative et l'assomption d'une position, mieux comprendre un pays sans le réduire à être « la république des mollahs » :

[SK] Elle sait que, comme elle, nous aimons beaucoup l'Iran, [...] l'Iran ça crée des liens. [...] ce qu'elle a vu de l'intérieur de l'Iran et ce qu'elle en a rapporté, constitue un témoignage exceptionnel et unique sur l'une des périodes les plus mouvementées de l'histoire de la république islamique. Elle sait que son récit peut aider à une meilleure compréhension d'un pays souvent présenté de manière caricaturale. $(1 / 2)^{2}$

[SK] Sur la question de savoir si Clotilde Reiss est un agent de la DGSE, n'attendez pas de « Les pieds sur Terre » la moindre réponse, ni même la moindre question. [...] Ce qui nous intéresse aujourd'hui en revanche, c'est ce qui se passe derrière la photo de son procès qui a fait le tour du monde. Quelle histoire cette photo raconte pour Clotilde et les autres prisonniers à côté d'elle? $(2 / 2)$

C'est aussi en aval de la diffusion que les émissions s'inscrivent dans le dialogisme interlocutif à la faveur de l'interactivité liée aux dispositifs en ligne, qui montrent et amplifient « l'impossible clôture des discours médiatiques » (Moirand, 2004). Les commentaires des auditeurs qui font suite aux émissions (prises de position, appréciations, fragments de témoignage en écho adressés aux producteurs, à C. Reiss, à la chaîne ou spécifiquement à tel ou tel internaute) sont

1 En dehors de la courte phrase de S Kronlund qui annonce le sujet de l'émission en fondu sonore avec le générique, l'auditeur est d'abord en présence de la voix et des paroles de C. Reiss. La présentation de la productrice n'intervient qu'ensuite avant de retrouver la narratrice.

$21 / 2$ renvoie à l'émission du 10 juin, $2 / 2$ à celle du 11 . $\mathrm{SK}=$ Sonia Kronlund. $\mathrm{CR}=$ Clotilde Reiss. 
toujours consultables sur le site de France-Culture. D'autre part l'émission et son équipe sont mentionnées, décrites ou critiqués dans d'autres organes de presse ou sur tel ou tel site d'opinion'1.

Les narrations des Pieds sur terre sont souvent des portraits, des autoportraits, tablant sur les personnes considérées dans leur singularité comme mode de connaissance. Lorsque S. Kronlund introduit la semaine sur l'Iran, elle insiste sur ce filtre de l'expérience individuelle non comme fin en soi mais comme accès à la compréhension du collectif. " C'est cette transformation (de la République islamique en dictature) que nous vous racontons donc cette semaine à la première personne », dit-elle au début de la série d'émissions.

Documentaire, reportage, témoignage, les trois caractérisations génériques sont présentes. Sans faire fi de la complexité des critères de distinction, on pourrait dire que documentaire renvoie à la forme radiophonique aussi bien qu'au point de vue organisateur, reportage à la relation à la réalité et au terrain, témoignage à la relation à la vérité et au statut énonciatif du locuteur.

Très généralement, le témoignage lie l'expérience à une éthique de l'authenticité : « Le témoin est donc celui dont la certification engage la personne, et qui en disant : 'j'y étais' et 'cela a été', déclare en même temps : 'voilà ce que j'ai été'. »(Amossy, 2004). La narration de C. Reiss est celle d'un témoin acteur et pas seulement spectateur, car elle a vécu de l'intérieur la situation des Iraniens, avec une grande proximité culturelle ${ }^{2}$, elle y était et elle peut dire ce qui a été :

[CR] Je vous ai ramené mes dessins parce que en sortant de prison j'avais un gros besoin de témoigner, mais l'écriture ce n'est pas vraiment mon truc, et en fait c'est encore plus des images que des mots qui m'ont marquée ( $1 / 2$, incipit)

1 Qu'il s'agisse de l'Iran ou de la radio. Par exemple France-Culture en général et les Pieds sur terre en particulier font l'objet de critiques très acerbes au motif notamment que la chaîne sacrifierait la culture ou favoriserait l'extrême-gauche avec ses documentaires : http://regardfc.forumn.net/regards-sur-les-emissions-fl/ les-pieds-sur-terre-t59-10.htm.

2 Cette proximité est sensible par exemple quand elle fait référence aux usages du quotidien (donnant le nom des sandalettes en persan), ou qu'elle cite en persan puis traduit une phrase du poète Molana. 
L'équipe de l'émission ne part pas en reportage ${ }^{1}$ sur le terrain pour observer et recueillir des discours, c'est la jeune femme qui vient à elle, qui vient à nous comme si elle avait fait un reportage " embarqué » et involontaire. Même si son récit suit le fil chronologique de son histoire, elle prend appui sur les dessins qui représentent les lieux et les personnes et marquent les étapes, des notes personnelles, des documents publics comme ce journal « composé de photos prises lors des manifestations » et « qui invite à la délation, à dénoncer des gens qu'on connaîtrait » (CR, 1/2).

La narration, très personnelle par les faits qu'elle évoque ${ }^{2}$, les émotions qu'elle décrit, les propos qu'elle rapporte, fait accéder à un vécu collectif, comme pour donner voix à ceux qui ne peuvent pas raconter, ses compagnons de détention ou de procès, d'autres encore, victimes anonymes, et les Iraniens en général :

[CR] Je ne parlerai pas de mes codétenues en fait. Je préfère pas, parce que oui j'ai peur pour elles $(1 / 2)$

$[\mathrm{CR}]$ là, il y a 4 pages dactylographiées pour expliquer tous mes méfaits ${ }^{3}$ et en fait [...] il y a trois pages qui sont les informations obtenues au cours des interrogatoires qui sont faits face à un mur, les yeux bandés avec trois, quatre hommes derrière toi qui te font peur, qui te forcent à dire les choses comme ils le veulent. [...] la justice iranienne c'est ça pour moi, mais aussi pour tous les Iraniens. Et pour moi, quand je vois ça, c'est ce qui me fait le plus mal au cœur.[SK]Et toi tu pensais que la justice iranienne, elle était juste ? [CR] En fait je ne l'avais pas vue dans une telle horreur. $(2 / 2)$

L'oralité radiophonique, et le mode de construction du récit, montrent le témoignage dans son élaboration, dans le temps nécessaire pour qu'il émerge, au premier chef pour la narratrice. Le déroulement narratif est globalement chronologique (de l'arrestation à la libération),

1 Mais l'émission (et les archives sur le site) classe(nt) les deux entretiens dans la catégorie documentaire en indiquant bien « Reportage : Sonia Kronlund, Bahar Makooi, Réalisation : Emmanuel Geoffroy ».

2 Par exemple quand elle raconte son anniversaire en prison en l'honneur duquel ses co-détenues et elle-même avaient gardé la ration de fruits de toute la semaine, qu'elle explique ce qu'elle pouvait voir de ses interrogateurs en ayant les yeux bandés (chaussures, mains), l'importance de leurs voix ou qu'elle évoque son entrevue avec un terrible procureur.

3 Il s'agit de l'acte d'inculpation. 
mais avec des récapitulations et des anticipations, des synthèses sur les événements qui se sont répétés (les interrogatoires), les bifurcations sur les cas particuliers, ou la mise en exergue de moments forts. Le cours du récit est à la fois structuré et libre, laissant apparaître le travail de mémoire, de réflexion et de conversion en mots d'un vécu. Le mouvement du discours semble être celui que la narratrice accomplit sur ellemême à partir de ces traces rapportées (les images, les phrases, les documents) pour comprendre et transmettre ce qui lui est arrivé. Des allers-retours caractéristiques d'un dire de soi se font du temps de l'histoire à celui de la narration, entre plusieurs « je » correspondant à des époques différentes, et d'une personne verbale à une autre (énallage du pronom de $2^{\mathrm{e}}$ personne et emploi du « on » comme sujet collectif quand il s'agit de faire état de l'expérience partagée de la détention / «je» pour se désigner comme sujet racontant ou dans sa situation particulière ) :

[CR] Et puis on est arrivé devant cette grande prison. Je me souviens aussi de la première image, je l'ai dessinée, c'était à l'extérieur de la prison où il y avait une foule de gens. J'ai compris ensuite que c'était les parents des prisonniers qui attendaient à l'extérieur d'avoir des nouvelles de leurs enfants. (1/2)

[CR] Alors en fait, on vient te chercher dans ta cellule, et ça c'est une angoisse terrible parce qu'on est dans une cellule, il ne se passe rien. On attend que la journée se passe et tout à coup il y a la gardienne qui vient et qui dit : « toi maintenant debout, tu te lèves, tu t'habilles, tu te prépares, tu as un interrogatoire. » Donc tu te lèves. $(1 / 2)$

La modalité conversationnelle de l'entretien, avec la simplicité d'un oral spontané sur une trame réfléchie, participe à cette dynamique à laquelle les auditeurs sont associés. La part du dialogue correspond à la manifestation d'une écoute puisque S Kronlund n'interrompt pas C. Reiss mais lui fait préciser un détail ou réagit sans régularité ou prévisibilité dans la fréquence de ses interventions. S. Kronlund permet une médiation avec les auditeurs qu'elle représente, mais sans s'assimiler à eux, puisqu'elle reste elle-même avec sa connaissance préalable de l'Iran et de son interlocutrice.

Le montage est l'une des caractéristiques qui permet de définir le documentaire radiophonique ${ }^{1}$ selon Deleu (Girard \& Deleu, 2009). Le

1 «Par documentaire, on suppose la définition suivante : enregistrement de sons, 
montage très soigné et précis de Les Pieds sur terre est perceptible sous plusieurs angles.

Une macro structuration intervient au niveau de la programmation générale et de ses choix, de la programmation des séries (ici sur une semaine), et de celle de chaque émission. Le triple incipit des deux émissions assure différents repérages fortement sémantisés. Premier incipit : l'annonce du sujet (une phrase en clôture fait pendant à cette annonce correspondant aux bornes de l'émission), suivie par le générique qui est lui-même un montage ${ }^{1}$, et dont le nappé musical porte l'énoncé du titre de l'émission par S. Kronlund. Deuxième incipit : C. Reiss parle directement pendant quelques minutes («Je vous ai apporté mes dessins[...] » (1/2), « Ça c'est le jour où j'ai été libéré, donc c'est la fameuse photo qui a fait le tour du monde $[\ldots] »(2 / 2)$. Troisième incipit : le discours de la productrice (cité supra) qui contextualise et assume une position ( « C'est la première fois qu'elle raconte publiquement ce qui lui est arrivé.... » (1/2) «Au nombre des milliers d'arrestations qui font déborder les prisons d'Iran depuis plus d'un an....» (2/2). Ensuite, le récit-conversation du témoignage suit son cours comme pour laisser au flux de la parole advenue son naturel, et respecter le temps de la narratrice et de la narration.

Cependant le récit n'est pas tout d'un bloc, il n'est pas sans interlocuteur et pas sans modulation. Une double respiration intervient, celle, interne du dialogue, celle, externe, des pauses sonores qui contribuent

montage, mixage selon une réalisation déterminée, dans des conditions qui ne sont pas celles du direct et n'entrent pas dans le cadre de journaux radiophoniques. C'est un genre assez rare à la radio ; on l'entend sur le service public et sur le web » (Girard \& Deleu, 2009). Deleu appelle " parole documentaire » la forme de «l'interview montée [où] le journaliste ou l'animateur donne la parole à une personne racontant une expérience » (Deleu, 2000).

1 La pièce musicale du groupe Troublemakers intitulée « Get Misunderstood » (2001) fait entendre des voix non chantant mais parlant, et intègre successivement un extrait de dialogue de film ( «Personne ne sait ce qui se passe aujourd'hui parce que personne ne veut qu'il se passe quelque chose »), puis un extrait de reportage au vif d'un affrontement de rues (mai 68 ?), puis un autre extrait de film (« Et pourquoi on le ferait pas plus souvent, on pourrait le faire tous les jours, dix fois par jour, vingt fois par jour »). Le premier extrait de film (J.-P. Léaud dans Liberté, La Nuit de $\mathrm{Ph}$. Garrel, 1983) provient d'un dialogue où il est question de révolution, le second vient de L'an 01 « On arrête tout, on réfléchit et c'est pas triste » (Gébé et J. Doillon, 1973). La présence de la voix, la revendication collective, ainsi que les thématiques du changement social et de l'engagement sont donc allusivement ou explicitement inscrites dans le générique. 
à une scénarisation discrète et qui fonctionnent connotativement, entre illustration, allusion et évocation. Ces pauses débutent ou se prolongent parfois sur fond de parole, et n'ont pas le caractère d'intermède d'autres programmes. Ce sont des musiques évoquant le Moyen Orient (instrument à cordes pincées - luth ? - ou musique plus orchestrée), chants parmi lesquels un de propagande dans laquelle on entend le nom de l'ayatollah Khomeyni, psalmodie «Allah akbar », et en clôture d'émission, chansons du monde anglo-saxon (The Sisters of mercy de L. Cohen -1/2-,une autre chanson que nous n'avons pas identifiée -2/2-). Les éléments vocaux et/ou musicaux sont pour la plupart en contiguïté géographique, culturelle, politique et religieuse avec l'Iran. Mais le lien avec le discours narratif peut être plus étroit : le chant à la gloire de Khomeyni fait écho à ce que raconte C. Reiss sur l'absence de livres en prison, à l'exception d'un livre faisant l'éloge de l'ayatollah, la chanson de Cohen prolonge un passage dédié à la solidarité entre les co-détenues.

La cohérence du propos est donc aussi celle de la distribution de la parole, du choix du tempo et de l'ambiance sonore. Mais ce qui importe surtout, plus que la structure, c'est ce qu'on pourrait appeler la texture orale, l'intonation, le phrasé, le timbre de la narratrice, celle d'une voix nue dont l'intimité n'est pas dérobée et qui manifeste la qualité d'une présence à soi et à l'autre. La dimension poétique est solidaire de la dimension politique du documentaire qui permet comme le dit F. Antoine d'aller « à la rencontre d'un univers » (Girard \& Deleu, 2009).

Pour conclure, les deux émissions diffèrent donc, en fonction de leur genre, de leur identité propre liée à l'identité des deux chaînes de Radio-France qui les diffusent, à celle de leurs producteurs et collaborateurs. Elles invitent bien leurs auditeurs à une (re)construction narrative, mais en sollicitant des types d'écoute différents.

Le premier régime d'intelligibilité est celui de la matinale qui vise le décryptage en s'appuyant sur la doxa. Les bulletins d'information particulièrement fonctionnent comme des réservoirs à histoires, et combinent saturation et allusion, en se fondant sur l'inférence et la mémoire.

Le second régime, celui du documentaire, fait confiance à l'expérience singulière et à la narration de l'intérieur, à la temporalité éprouvée qui appelle une écoute d'immersion. Il vise à exprimer l'expérience et à lui donner un sens, tourné vers l'action personnelle ou sociale. Ecouter 
pour entendre, pour comprendre et pour agir, pour vouloir qu'il se passe quelque chose.

\section{Références}

Amossy, R. (2004). L'espèce humaine de Robert Antelme ou les modalités argumentatives du discours testimonial. Semen, 17. Disponible à : http://semen. revues.org/2362.

Castant, A. (1995). Littérature et télécommunication: vertiges du vite et le monde sonore. In Hermès sans fil: imaginaire de la communication à distance. ClermontFerrand : Association des publications de la Faculté des lettres et sciences humaines.

Charaudeau, P. (Ed.) (1984). Aspects du discours radiophonique. Paris : Didier Erudition.

Charaudeau, P. (2005). Les médias d'information. L'impossible transparence du discours. Bruxelles : De Boeck-INA.

Deleu, C. (2000). Le documentaire radiophonique : un genre marginal... plein d'avenir. Les Cahiers du journalisme, 7, pp. 146-195.

Girard, J., \& Deleu, C. (2009). Dynamiques contemporaines du documentaire radiophonique. Disponible à : http://www.grer.fr/upload/articles_en_ligne/ Dynamiques_contemporaines_du_documentaire_radiophonique_compte-rendu_ du_seminaire_du_31_mai_2008.pdf.

Moirand, S. (2004). L'impossible clôture des corpus médiatiques. Tranel, 40, pp. 71-92. Mouillaud, M. (1984). Espace et temps radiophonique. In Charaudeau P. (Ed.) Aspects $d u$ discours radiophonique. pp. 61-70. Paris : Didier Erudition

Tétu, J.-F. (1994). La radio, et la maîtrise du temps, Études de communication, 15. Disponible à : ligne http://edc.revues.org/2684

Tétu, J.-F. (1993). L'actualité ou l'impasse du temps, in Bougnoux, D. (Ed.) Textes fondamentaux des sciences de la communication. pp. 714-722. Paris : Larousse. 